\title{
CONDUCTIVITY OF OPTICALLY EXCITED ELECTRONS IN GaAs IN QUANTIZING MAGNETIC FIELDS*
}

\author{
J. Eusakowski, M. Grynberg \\ Institute of Experimental Physics, University of Warsaw \\ Hoża 69, 00-681 Warszawa, Poland \\ AND S. HUANT \\ High Magnetic Field Laboratory, MPIF and CNRS \\ 25, Avenue des Martyrs, 38042 Grenoble, France
}

\begin{abstract}
Magnetoconductivity $(\sigma)$ measurements on an $n$-type molecular beam epitaxy grown epitaxial layer and on a bulk liquid encapsulated Czochralski grown undoped semi-insulating $\mathrm{GaAs}$ samples were performed for magnetic fields $(B)$ up to $21 \mathrm{~T}$ at $4.2 \mathrm{~K}$. To enable current measurements in a wide range of $B$ both samples were permanently illuminated with a band-to-band light. It is shown that for sufficiently high magnetic fields $\sigma(B)$ dependence is the same for both materials. This result underlines a role of scattering by long-range fluctuations of the electrostatic potential in high-quality $n$-GaAs in quantizing magnetic fields.
\end{abstract}

PACS numbers: $72.20 . \mathrm{Hy}, 72.20 . \mathrm{Ht}$

\section{Introduction}

Fluctuations of the electrostatic potential arise in semiconducting materials due to a random distribution of charged centres [1]. The amplitude of fluctuations grows with an increase in concentration of localized charges $N_{l}$ and with a decrease in concentration of screening carriers $N_{\mathbf{s}}$. In degenerate materials screening is accomplished by free carriers, which concentration is much greater than that of localized ones. However, in compensated materials $N_{l}$ is usually orders of magnitude greater than $N_{\mathbf{s}}$, particularly at low temperatures, and the amplitude of fluctuations may attain a large value.

Long-range fluctuations of the electrostatic potential influence properties of a semiconductor. Recent magnetoconductivity and magneto-optical studies of

*This work is partially supported by the State Committee for Scientific Research (Republic of Poland) grant No. 202659101. 
semi-insulating (SI) GaAs at liquid helium temperatures have shown such influence on optical properties and the electron transport in this material [2]. In particular, fluctuations were found to be responsible for observed magnetic field induced localization of hot electrons [3] and an increase in probability of autoionization of excited states of shallow donors [4]. On the other hand, high quality $n$-type materials with a low concentration of dopants and a low compensation are usually regarded as free of this type of fluctuations, because one expects that the condition $N_{l} \ll N_{\mathrm{s}}$ is satisfied even at low temperatures. However, results which are presented in this paper show that in a high magnetic field one should expect an essential growth of fluctuations even in such materials.

\section{Experiment}

Conductivity measurements were performed on two GaAs samples at $4.2 \mathrm{~K}$ for magnetic field up to $21 \mathrm{~T}$. One of the samples was an MBE (molecular beam epitaxy) epitaxial layer with a concentration of shallow donors and a compensation equal to about $10^{14} \mathrm{~cm}^{-3}$ and 0.2 , respectively. The other sample was cut from a LEC (liquid encapsulated Czochralski) wafer of undoped SI GaAs with the EL2 as the main deep level, which concentration was equal to $5.3 \times 10^{16} \mathrm{~cm}^{-3}$. In the SI material a residual concentration of shallow donors and acceptors is expected to be of the order of $10^{15} \mathrm{~cm}^{-3}$. In this material, at liquid helium temperature the Fermi level is pinned to the EL2 which leads to a total absence of free carriers. To enable current measurements such sample was illuminated with a laser light ( $\lambda=0.4866 \mu \mathrm{m}$; this illumination was also used in the case of the $n$-type sample) which corresponded to band-to-band excitations. The measurements of the SI sample were performed after bleaching the EL2 to the metastable state [5] which was then inaccessible to ionization by light. The bleaching process neutralizes all the EL2 centres which means that the sample can be considered as a compensated semiconductor with shallow donors and shallow acceptors as the dominant charged centres. From the point of view of a spatial distribution of charges in an investigated material the applied experimental procedure brought the SI sample to be much similar to the $n$-type one.

Optical excitations generate both free electrons and holes. However, the hole mobility in GaAs is about 10 times smaller than that of an electron and in the following the current will be regarded as carried by electrons only. Free electrons are generated in a very thin layer at the surface of a sample but they diffuse into the bulk due to a large gradient of a concentration and some of them are captured by shallow donors, which was verified by magneto-optical experiments [6].

The laser light was focused on an optic fiber which ended a few millimeters above a sample surface. Each sample was supplied with four $\mathrm{Au} / \mathrm{Ge} / \mathrm{Ni}$ contacts (see the inset in Fig. 1). The electrical arrangement consisted of a sample connected in series with a voltage source and an ammeter. Two outer contacts ( $a$ and $d$ ) were used for supplying a current to a sample and the two inner ones ( $b$ and $c$ ) for measuring a voltage drop in the middle part of a sample. The measurements were performed in a Bitter magnet with the magnetic field perpendicular to the applied electric field. 


\section{Results and discussion}

Figure 1 shows the results of $n$ easurements performed on the $n$-type sample. When the sample was not illuminated, current measurements were possible only for $B$ up to about $6 \mathrm{~T}$. For higher $B$ freezing of free electrons caused an increase in the resistance of the sample above $10^{10} \Omega$ which disabled measurements because of a too low resolution of the ammeter. For small magnetic fields $(B<2 \mathrm{~T})$ impact ionization of shallow donors was observed which resulted in a sharp increase in the current by a few orders of magnitude. An increase in the magnetic field leads to an increase in the threshold electric field for impact ionization. Thus, a strong decrease in the current is observed if it is monitored as a function of $B$ for a constant voltage measured between the $b$ and $c$ contacts. Impact ionization and its vanishing with an increase in $B$ was also observed when the sample was illuminated. In this case, however, a decrease in the current with an increase in $B$ was much smaller.

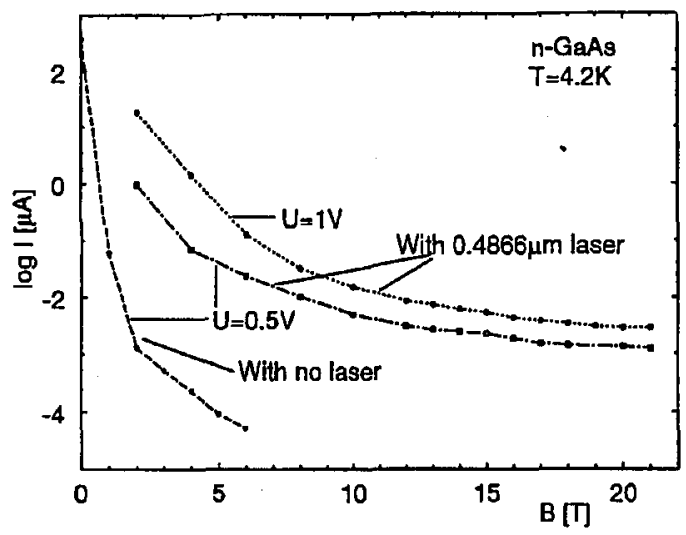

Fig. 1. Current $(I)$ vs. magnetic field $(B)$ for the $n$-type MBE layer for the indicated voltages measured between the contacts $b$ and $c$. The inset shows a schematic diagram of the electrical circuit.

Figure 2 shows the results of measurements for the SI sample. For low voltages no impact ionization was observed and the increase in the magnetic field up to $20 \mathrm{~T}$ caused a decrease in the current by about two orders of magnitude. For larger voltages impact ionization of shallow bound states (neutral donors and electrons bound in wells of the fluctuating potential) takes place. An increase in $B$ causes electron localization which is the reason of a strong current decrease for $3 \mathrm{~T}<B<6 \mathrm{~T}$. This process was described in details elsewhere [2,3]. In the localization regime the shape of the current on magnetic field curve does not depend on whether the impact ionization took place or not.

Current on magnetic field dependence for the two samples for small $B$ shows a pronounced difference. For the $n$-type not illuminated material it monotonically decreases with an increase in $B$ while for the SI sample a broad maximum is observed for $B \approx 2 \mathrm{~T}$. This difference may result from the fact that for the $n$-type 


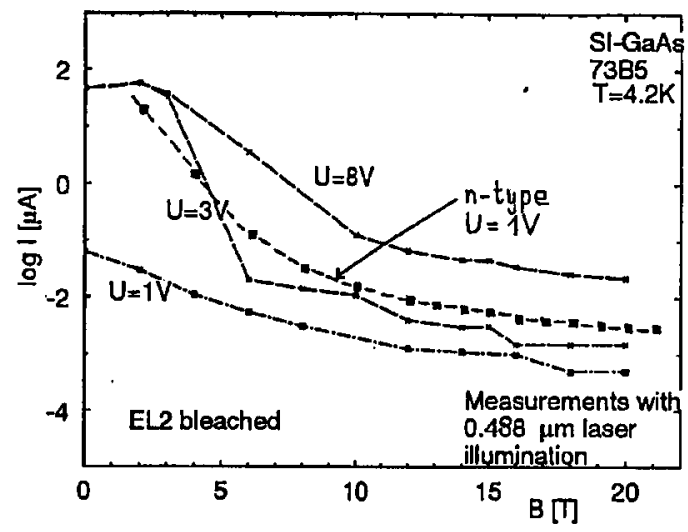

Fig. 2. Current $(I)$ vs. magnetic field $(B)$ for the SI GaAs LEC sample for the indicated voltages measured between the contacts $b$ and $c$. One of the curves shown in Fig. 1 is also presented here for comparison.

sample electrons are freezed out on shallow donors, while for the SI case they are scattered and bound by fluctuations of the electrostatic potential. Thus a mechanism which causes localization of hot electrons in both cases is different.

However, an influence of the magnetic field on the current for high magnetic fields is the same for both materials. Since free carriers are generated at the surface of the samples one may consider that this similarity is caused by a surface conductivity. However, in such case $I(B)$ curve for small $B$ should also show the same shape for both materials, which is not observed. What is more, the maximum on the $I(B)$ curve was also observed for a subband illumination of the SI sample, which generated free electrons in the bulk (a result not presented here). Thus one can argue that under the band-to-band illumination a dominant part of the current is carried in the bulk of the investigated samples.

From previous studies [2, 3] it is known that in SI GaAs scattering by potential fluctuations plays a dominant role in a hot-electron conductivity. The presented results thus reveal that this type of scattering may be also important in high quality materials. The reason for this is a freeze-out of free carriers which strongly reduces a concentration of carriers which screen potential fluctuations. In the present experiment the freeze-out was achieved by applying quantizing magnetic fields which localised free electrons due to an increase in the shallow donor binding energy [7] and a density of localised states at the bottom of the conduction band [8]. However, similar effects should be also observed at very low temperatures.

\section{Acknowledgments}

The authors are thankful to Dr. E. Kamińska and Dr. A. Piotrowska, Institute of Electron Technology, Warsaw, for preparing contacts to the samples. 


\section{References}

[1] B.I. Shklovskii, A.L. Efros, Electronic Properties of Doped Semiconductors, Springer-Verlag, Berlin 1984.

[2] J. Lusakowski, K. Karpierz, M.L. Sadowski, M. Grynberg, Solid State Commun. 84, $231(1992)$.

[3] J. Łusakowski, R. Merten, M. Grynberg, Acta Phys. Pol. A 82, 551 (1992).

[4] K. Karpierz, J. Eusakowski, M.L. Sadowski, M. Grynberg, Physica B 184, 403 (1993).

[5] M. Kamińska, Rev. Phys. Appl. 23, 793 (1988).

[6] M.L. Sadowski, K. Karpierz, M. Grynberg, Phys. Rev. B 34, 7332 (1991).

[7] W. Rösner, G. Wunner, H. Herold, H. Ruder, J. Phys. B, At. Mol. Phys. 17, 29 (1984).

[8] M.I. Dyakonov, A.L. Efros, D.L. Mitchell, Phys. Rev. 180, 813 (1969). 